
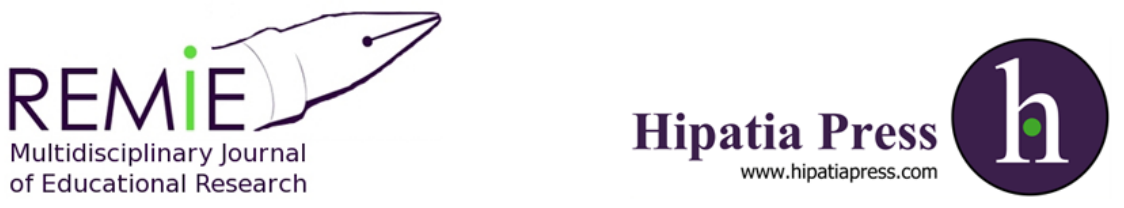

Instructions for authors, subscriptions and further details:

http://remie.hipatiapress.com

\title{
Formación de Familiares y Voluntariado. Los Beneficios de la Solidaridad en la Escuela
}

Carme Garcia Yeste ${ }^{1}$, Laura Ruiz Eugenio², Miquel Àngel Comas ${ }^{3}$

1) Universitat Rovira i Virgili. España

2) Universitat de Barcelona. España

3) Universidad Internacional de La Rioja. España

Date of publication: June $15^{\text {th }}, 2019$

Edition period: June 2019- October 2019

To cite this article: García, C., Ruiz, L., Comas, M.A. (2019). Formación de Familiares y Voluntariado. Los Beneficios de la Solidaridad en la Escuela. Multidisciplinary Journal of Educational Research, 9(2), 144-168. doi:

10.17583/remie.2019.4188

To link this article: http://dx.doi.org/10.17583/remie.2019.4188

\section{PLEASE SCROLL DOWN FOR ARTICLE}

The terms and conditions of use are related to the Open Journal System and to Creative Commons Attribution License (CC-BY). 


\title{
Formación de Familiares y Voluntariado. Los Beneficios de la Solidaridad en la Escuela
}

Carme Garcia Yeste

Universitat Rovira $i$ Virgili

Miquel Àngel Comas

Universidad Internacional de la Rioja

\author{
Laura Ruiz Eugenio \\ Universitat de Barcelona
}

\begin{abstract}
Resumen
El artículo tiene por objetivo analizar los beneficios de la solidaridad promovida por el voluntariado en la formación de familiares en diversas escuelas, de educación infantil y primaria en España, que son Comunidades de Aprendizaje. Uno de los principios comunes de la organización de estas escuelas es la solidaridad que se concreta en diversas actuaciones. Entre estas, la inclusión de personas voluntarias de muy diversos perfiles en la formación de familiares. Los resultados que se presentan son parte del estudio más amplio, 'La mejora del sistema educativo a través de la formación de familiares de grupos vulnerables' (2014-2017). Se ha llevado a cabo trabajo de campo cualitativo en 8 escuelas (entrevistas semiestructuradas, grupos de discusión y relatos de vida) con familias, alumnado, profesorado y voluntariado, de cinco comunidades autónomas. Entre los principales resultados se constata que el voluntariado contribuye a fortalecer las dinámicas solidarias en estas escuelas y a mejorar el clima escolar. Concretamente 1) creando más entornos de aprendizaje donde se establecen vínculos de ayuda mutua entre estos y las familias y entre las propias familias, 2) creando interacciones igualitarias que fomentan la amistad y la confianza, 3) aumentando la motivación y las expectativas de las familias por su formación. La inclusión de voluntariado, especialmente en escuelas de entornos desfavorecidos, está promoviendo formación de familiares que está contribuyendo a la mejora del aprendizaje y la motivación no solo de los y las familiares sino también de los niños y niñas. Estos resultados ofrecen más evidencias a las ya existentes sobre factores que contribuyen a la superación del determinismo educativo que establece que el logro académico está determinado por el nivel socioeconómico y educativo de las familias.
\end{abstract}

Palabras clave: voluntarios, formación de familiares, comunidad, entornos de aprendizaje, interacciones, capital social, justicia social 


\title{
Family Education and Volunteering: The Benefits of Solidarity in Schools
}

\author{
Carme Garcia Yeste \\ Rovira i Virgili University
}

Miquel Àngel Comas

International University of La Rioja

\author{
Laura Ruiz Eugenio \\ University of Barcelona
}

\begin{abstract}
The aim of this article is to analyze the benefits of solidarity promoted by volunteering in family education at various Spanish elementary schools which are Learning Communities. One of the common organization principles in these schools is solidarity, which is visible in various spaces and actions. Among these, volunteers of very diverse profiles are included in family education. The results presented here are part of a broader research project, "The improvement of the educational system through the family education in vulnerable groups' (2014-2017), funded by the State Plan for Scientific and Technical Research and Innovation of the Government of Spain. Qualitative fieldwork has been carried out in eight schools (semistructured interviews, focus groups and life stories) with families, students, teachers and volunteers from five Autonomous Communities. Among the main results, it was highlighted that volunteering contributes to strengthening the solidarity dynamics in these schools and to improving the school climate. Specifically, 1) creating more learning environments where mutual help relationships are established between volunteers and families as well as between families, 2) creating egalitarian interactions that foster friendship and trust, 3 ) increasing motivation and expectations of the families' training. The inclusion of volunteers in family education, especially in schools in disadvantaged environments, is contributing to the improvement of learning and motivation not only in family members, but also in children. These results offer more evidence to that already existing on factors contributing to the overcoming of educational determinism. This establishes that academic achievement is determined by the socioeconomic and educational level of families.
\end{abstract}

Keywords: volunteers, family education, community, learning environment, interactions, social capital, social justice 
C a solidaridad es uno de los principios de la organización en escuelas que están implementando actuaciones educativas de éxito como Comunidades de Aprendizaje (Aubert et al., 2008). Esta solidaridad se hace patente tanto en la organización de la escuela como en cada una de las actuaciones educativas que se implementan. Entre estas actuaciones se encuentra la formación de familiares. La comunidad científica internacional desde hace décadas ha demostrado que las interacciones que el alumnado tiene en su contexto sociocultural son clave en su rendimiento escolar (Bonell y Ríos, 2014; Garcia-Carrion, Molina-Luque y Molina-Roldan, 2018). Más allá de las interacciones que tienen con el profesorado, los niños y niñas cada vez aprenden más de las interacciones que tienen con otras personas dentro y fuera de la escuela, especialmente con sus familiares, pero también con otras personas referentes en la comunidad (Dearing, Kreider, Simpkins y Weiss, 2006; Epstein y Sheldon, 2002). Para que esas interacciones tengan una influencia positiva en los resultados académicos de los niños y niñas, no solo es necesaria la formación del profesorado sino también de las familias (Jasis, Ordoñez y Jasis, 2012; Epstein y Sanders, 2006). Las investigaciones internacionales también han dado evidencia de que los resultados académicos de los niños y las niñas no están determinados por el nivel educativo de las familias, sino que estos mejoran cuando sus familias también se están formando, especialmente aquellas familias no académicas y pertenecientes a minorías culturales (Flecha y Soler, 2013). En las escuelas que están implementando actuaciones educativas basadas en estas evidencias científicas e implican a perfiles muy diversos de personas voluntarias, se están promoviendo interacciones solidarias que contribuyen a llevar a cabo una formación de familiares que responde a los intereses y necesidades de las familias (Diez, Gatt y Racionero, 2011).

En este artículo se presentan resultados del análisis de la percepción de familiares, profesorado y voluntariado sobre los beneficios de la solidaridad en las interacciones que se crean en la formación de familiares entre el voluntariado y las familias. Concretamente, se analizan los beneficios de las dinámicas solidarias promovidas por el voluntariado en formación de familiares en ocho escuelas de educación infantil y primaria en diferentes territorios del estado español. Los resultados que aquí se presentan son parte de un estudio más amplio sobre 'La mejora del sistema educativo a través de 
la formación de familiares de grupos vulnerables' (2014-2017). Mostrando así a su vez el potencial social y pedagógico que tienen los centros educativos transformados en comunidades de aprendizaje. Dónde no solo existe una transformación del propio centro (tanto a nivel de resultados académicos como de convivencia) si no del entorno cercano como son las familias de los niños y niñas y los vecinos/as del barrio.

Para ello, en un primer apartado, se presentan aportaciones de la revisión de la literatura sobre el impacto de la solidaridad en la educación y en la formación de familiares, así como el rol del voluntariado. A este apartado, le sigue el de metodología que visualiza las técnicas de recogida de la información implementadas y los criterios de selección de las escuelas participantes. A continuación, el apartado de resultados es expuesto seguido de unas conclusiones y la implicación social de estos resultados.

\section{Marco Teórico}

La literatura internacional sobre los beneficios de la solidaridad en la escuela está aumento en los últimos años. Si es verdad que en el ámbito del estudio de las organizaciones, concretamente sobre las cooperativas como tipología de empresa, se ha tenido más en cuenta (Cheney et al., 2014; Flecha y Ngai, 2014), en otros ámbitos como el educativo es más reciente. Algunas de estas referencias se pueden encontrar en el estudio del voluntariado como activo intangible en ONGs (Youngblood, 2017), en los actos de ayuda informal como comportamiento prosocial y compromiso cívico (Hermansen, 2016), en las relaciones intergeneracionales entre los hijos e hijas de inmigrantes y sus familias en el proceso de transición de casa a la escuela (McGovern y Devine, 2016), en la creación de lazos de solidaridad entre el profesorado y estudiantes como práctica de éxito en aulas multiculturales (Boucher, 2016), en las interacciones en entornos de aprendizaje como las tertulias literarias dialógicas (Garcia, Gairal, Munte y Plaja, 2018; Llopis, Villarejo, Soler, y Álvarez, 2016; Pulido-Rodriguez, Amador, y Alonso Rodrigo, 2015) y en grupos interactivos (Aubert, Molina, Shubert, y Vidu, 2017; Valero, Redondo-Sama y Elboj, 2018), en actuaciones educativas para la prevención de la violencia de género (Pulido, Elboj, Campdepadros y Cabre, 2014) y en 
los movimientos sociales y educativos impulsados por mujeres de base o pertenecientes o minorías étnicas (Sorde, Serradell, Puigvert y Munte, 2013).

En el marco de estas contribuciones, en la última década también se han recogido evidencias sobre dinámicas solidarias que forman parte de actuaciones educativas que a la vez que son eficaces, generan equidad mejorando los resultados académicos de los hijos e hijas de familias de minorías culturales y en entornos desfavorecidos (Ramis-Salas, 2015). La Comisión Europea, manifestó en el documento Efficiency and Equity (Comisión Europea, 2006) la importancia de conocer cuáles son las actuaciones educativas eficientes y equitativas para su contribución a un crecimiento inteligente, sostenible e inclusivo de Europa. En este contexto, la superación de las desigualdades en los resultados académicos y el éxito educativo de los colectivos más vulnerables sigue siendo un reto para España y Europa (Comisión Europea, 2010). Es en este marco donde el proyecto de investigación INCLUD-ED. Strategies for inclusión and social cohesion in Europe from Education (2006-2011) identificó actuaciones educativas que estaban generando tanto equidad como eficiencia (Flecha, 2015). INCLUDED fue la investigación sobre educación escolar de más recursos y mayor rango científico de los Programas Marco de Investigación de la Comisión Europea hasta aquel momento; coordinado por un consorcio de 15 universidades de 14 países europeos diferentes, implicando a más de 100 investigadores e investigadoras. Esta investigación ha sido la única en ciencias sociales que la Comisión Europea ha incluido en la lista de las 10 mejores investigaciones científicas europeas de los últimos tiempos (European Commission, 2011). Este proyecto actualizó el listado de actuaciones educativas de éxito que se ha traducido a todas las lenguas de los estados miembros. A lo largo de cinco años, se revisaron las principales teorías y aportaciones científicas para la mejora del aprendizaje y la cohesión social desde la escuela y la comunidad, las reformas educativas de todos los países de la UE y las prácticas llevadas a cabo en centros educativos que, a pesar de encontrarse en contextos con dificultades, conseguían éxito educativo, tanto en resultados escolares como en convivencia (Flecha, 2017).

La formación de familiares, especialmente con grupos vulnerables, que responde a las necesidades e intereses de las propias familias es una de estas actuaciones educativas de éxito (Garcia-Yeste, Morla y Ionescu, 2018). Esta 
es una de las actuaciones que contribuye a superar el determinismo educativo que promulgaba el conocido Informe Coleman et al. (1966) y que habían analizado los autores de la reproducción social en el último cuarto del siglo XX. Autores como Baudelot y Establet (1976), Bowles y Gintis (1985) o Bourdieu y Passeron (1996), afirmaban que la escuela reproducía las desigualdades porque los niños y las niñas de los grupos más desfavorecidos eran los que obtenían los peores resultados. Pero ya durante el mismo periodo y hasta la actualidad muchas son las evidencias que se han recogido desde la investigación educativa que demuestran que se están implementando actuaciones que revierten ese determinismo, transformando el capital cultural y social de estos niños y niñas y de sus familias (Edmonds, et al., 1973; Epstein, 1996; Epstein, 2016; Garcia-Yeste, Morla y Ionescu, 2018).

Actualmente, miles son las escuelas que están implementando formación de familiares siguiendo esas evidencias científicas, entre ellas, las Comunidades de Aprendizaje en España, Italia, Portugal, Reino Unido, Malta, Chipre (https://seas4all.eu; https://utopiadream.info) México, Argentina, Chile, Brasil, Colombia, Ecuador y Perú (https://www.comunidaddeaprendizaje.com.es). Algunas de estas escuelas se han coordinado con diferentes agentes de la comunidad para poder llevar a cabo formación de familiares, conseguir voluntariado y trabajar conjuntamente con asociaciones de familiares, educadores y educadoras sociales, técnicos de inserción laboral, asociaciones de vecinos y vecinas, otras entidades del barrio y representantes de la administración pública, entre otros. En estas escuelas, las dinámicas solidarias forman parte tanto de la organización como de las interacciones que se dan en cada uno de los entornos de aprendizaje que se crean (Garcia-Carrion, Girbes, y Gomez, 2015). Uno de estos entornos de aprendizaje es la formación de familiares donde se incluye la participación de voluntariado (Aubert, et al. 2008). A su vez, las familias también participan en otros entornos de aprendizaje con los niños y niñas, por ejemplo, en los grupos interactivos en las aulas o en la biblioteca tutorizada (Garcia-Carrion, et al., 2018).

Como es sabido, los niños y las niñas no aprenden tanto de lo que dicen las personas adultas sino de lo que hacen. Estas escuelas educan en valores como la solidaridad porque trasforman toda su actividad en torno a ese valor. No se limitan a hablar de valores como la solidaridad o tienen programas 
sobre solidaridad, sino que todas sus actividades y todas las interacciones que se dan en ella se basan en la solidaridad. Los niños y las niñas ven como hay personas voluntarias que están implicadas en la formación de sus familias, pero también ven como sus familiares les ayudan en los grupos interactivos o en la biblioteca tutorizada. En actuaciones como grupos interactivos se educa en solidaridad, aunque no se hable de solidaridad, porque los niños y las niñas, las familias y las personas voluntarias lo ponen en práctica cada día. Aprenden que la solidaridad no es algo sólo de lo que hay que hablar, sino que es algo útil porque contribuye al éxito de todos y todas (De Botton, 2015).

Los efectos de estas dinámicas solidarias no sólo se observan en la mejora de los resultados académicos sino también en la mejora de las relaciones entre los niños y las niñas y, en las relaciones entre el profesorado y las familias, así como se traslada al entorno fuera de la escuela, contribuyendo a crear un clima de solidaridad y amistad también en la comunidad (Flecha y Soler, 2013). El voluntariado es parte de esas dinámicas solidarias y su implicación en estas escuelas se rige por un código ético y contrato de colaboración. Las personas voluntarias se comprometen a tener altas expectativas hacia los niños y las niñas y sus familias. La diversidad de los perfiles del voluntariado enriquece la experiencia y el conocimiento que estos y estas aportan. Entre las personas voluntarias se encuentran los propios familiares (madres, padres, abuelos, abuelas, hermanos y hermanas mayores, etc.), recogiendo así la diversidad socioeconómica y cultural de cada escuela. Entre el voluntariado también hay estudiantado y profesorado universitario, personas de otras entidades del barrio o amigos y amigas. Estas personas se implican comprometiéndose con la finalidad última del proyecto que es contribuir al éxito educativo de los niños y niñas y a la mejora de la convivencia. Las personas voluntarias se pueden implicar en estas escuelas de maneras muy diversas, desde dinamizar las interacciones entre los niños y las niñas para que se ayuden a resolver las actividades en los grupos interactivos de matemáticas, lengua o inglés, hasta en la formación de familiares como clases de alfabetización, castellano oral para familias extranjeras, tertulias literarias dialógicas o aportando su conocimiento para desarrollar alguna actividad específica, como por ejemplo, formación sobre 
tecnologías de la información y la comunicación (Padros y Flecha, 2014; Valls y Kyriakides, 2013).

Los resultados que aquí se presentan contribuyen a complementar las evidencias ya existentes sobre los beneficios de estas dinámicas solidarias que se configuran y que también promueve el voluntariado, en este caso, en la formación de familiares.

\section{Metodología}

Se ha llevado a cabo trabajo de campo cualitativo a lo largo de los tres años de duración de la investigación en ocho escuelas de educación infantil y primaria de cinco Comunidades Autónomas (Andalucía, Castilla y León, Cataluña, Comunidad Valenciana y País Vasco). Los criterios para la selección de las escuelas han sido cinco: (1) escuelas con familias y alumnado pertenecientes a grupos vulnerables como minorías étnicas y de origen inmigrante; (2) escuelas que lleven más de dos años implementando formación de familiares; (3) escuelas de diferentes Comunidades Autónomas, asegurando diversidad territorial; (4) escuelas con evaluaciones y/o publicaciones científicas que aportan evidencias del impacto de la formación de familiares en la mejora educativa del alumnado y (5) diversidad en la oferta de formación de familiares ofrecida.

Este estudio se ha desarrollado desde la metodología comunicativa. Ello implica que las personas directamente afectadas por esta investigación, las familias participantes en la formación de familiares, el profesorado, el alumnado y el voluntariado, han participado en todo el proceso de desarrollo desde un diálogo igualitario con las personas investigadoras (Gomez, Puigvert, y Flecha, 2011). El uso de la metodología comunicativa en investigación ha demostrado obtener un elevado impacto tanto social, político como científico. Su objetivo vas mucho más allá de la simple descripción de la realidad para convertirse en si misma en a una herramienta de transformación que genere impacto social, y por tanto la mejora de las vidas de las personas participantes en las investigaciones (Gómez, Padrós, Ríos, Mara, \& Tekepuke, 2019). Esto implica que desarrollar nuestra investigación con dicha metodología y desde una orientación comunicativa supone contribuir desde el diálogo con los colectivos participantes en la 


\section{Garcia, Ruíz, \& Comas -Formación de Familias y Voluntariado}

investigación, y en este caso concreto, al conocimiento de la aportación que realiza el voluntariado en centros educativos que son comunidades de aprendizaje.

Los grupos de discusión, los relatos de vida y las entrevistas semiestructuradas de orientación comunicativa difieren de las técnicas tradicionales porque se establece un diálogo en igualdad entre la persona investigadora y la persona participante, desde el diseño de los guiones hasta el análisis de la información. La orientación comunicativa implica que existe una realidad natural objetiva y una realidad social construida socialmente que dependerá de los significados que se les atribuyan es por ello por lo que el diálogo se convierte en una herramienta indispensable en la creación de dichos significados (Gómez, Latorre, Sánchez, y Flecha, 2006). La persona investigadora aporta al diálogo el conocimiento científico previo sobre los temas objeto de estudio y la persona participante aporta el conocimiento de su mundo de la vida. Las interpretaciones de la realidad que se consideran cómo válidas son fruto de un consenso entre personas investigadoras y participantes. Este es el punto de partida para asegurar que existe un diálogo igualitario, donde se busca la situación ideal de diálogo rompiendo con el desnivel epistemológico ubicando en el mismo nivel a personas investigadoras e investigadas (participantes de la investigación), tanto en el proceso de la investigación como en el desarrollo e interpretación de los resultados.

El trabajo de campo ha constado de 95 entrevistas semiestructuradas con orientación comunicativa, 36 grupos de discusión comunicativos y 13 relatos de vida comunicativos. El siguiente cuadro recoge los perfiles de las personas participantes en cada tipo de técnica de recogida de la información implementada.

Como se ha señalado previamente este artículo presenta parte de los resultados de un proyecto más amplio. La pregunta de investigación de la que se recogen aquí los resultados es: ¿Qué beneficios aporta el voluntariado en la formación de familiares? Para este artículo se ha recogido exclusivamente la percepción de familiares, profesorado y voluntariado. No se recoge aquí la percepción de los niños y niñas, que ha sido objeto de otras contribuciones en proceso de publicación; no porque sean contradictorias a los resultados 
que se presentan aquí sino porque son análisis complementarios desde diferentes perspectivas.

Tabla 1

Numero de técnicas de recogida de la información por tipo y perfiles (2013-2016)

\begin{tabular}{llll}
\hline Tipo & Perfiles & Número & Total \\
\hline Entrevistas semiestructuradas con & Familias: & 48 & 95 \\
orientación comunicativa & Alumnado: & 12 & \\
& Profesorado: & 13 & \\
& Otros/as: & 22 & \\
\hline Grupos de discusión & Familias: & 19 & 36 \\
comunicativos & Alumnado: & 7 & \\
& Profesorado: & 5 & \\
& Otros/as: & 5 & \\
\hline Relatos de vida comunicativos & Familias: & 13 & 13 \\
& Alumnado: & 0 & \\
& Profesorado: & 0 & \\
& Otros/as: & 0 & \\
& &
\end{tabular}

Fuente: proyecto I+D EDUFAM

En los guiones de las diversas técnicas de recogida de la información, esta pregunta se trató a través de diversos temas: 1) rol y compromiso del voluntariado en la formación de familiares; 2) el tipo de relación entre las personas participantes en la formación de familiares y las personas voluntarias; 3) ambiente promovido por el voluntariado; 4) comparación del ambiente de esa formación de familiares con otro tipo de formación para personas adultas en las que haya podido participar; 4) relación del voluntariado con la mejora del aprendizaje; 5) relación del voluntariado con la motivación y expectativas sobre la formación de las propias familias; 6) relación del voluntariado con la mejora de la relación familia-escuelacomunidad.

En cada una de las técnicas de recogida de la información, según el perfil de la persona o personas participantes, estos temas eran tratados desde diferentes perspectivas y matices. En los grupos de discusión comunicativos, 
un grupo natural, por ejemplo de profesorado de una misma escuela, de alumnado hijos/hijas (de una misma clase) de participantes en formación de familiares, o de familiares que comparten una misma actividad de formación, elaboran una interpretación colectiva de los beneficios del voluntariado en la formación de familiares a través de un diálogo igualitario, evitando que la interpretación quede sometida a la imposición de unas opiniones sobre otras y estableciendo conclusiones consensuadas. Los relatos de vida comunicativos, a diferencia de los relatos de vida tradicionales, no son una narración biográfica interpretada por la persona investigadora, sino que son diálogos reflexivos sobre aspectos del mundo de la vida de la persona participante en el que la persona investigadora aporta conocimiento académico a esa reflexión desde una posición de igualdad.

Las categorías de análisis generales establecidas por el proyecto fueron cuatro: 1) beneficios en los y las familiares, 2) beneficios en el alumnado, 3) beneficios en el centro educativo y 4) beneficios en el barrio/comunidad. Para el estudio específico de los beneficios relacionados con el voluntariado en la formación de familiares se incluyó la variable transversal de "voluntariado" en cada una de estas categorías. La codificación y análisis de la información no se ha llevado a cabo a través de ningún programa informático. Se ha codificado la información extraída directamente de la transcripción total de todas las técnicas de recogida de la información en base a esas categorías de análisis. Las citas literales seleccionadas como evidencias que se presentan en este artículo son representativas de los resultados consensuados conjuntamente entre el equipo investigador y las personas participantes. Estas citas están identificadas con los códigos con C1, C2 (...) C8 según el centro, con E para las entrevistas, con GD para los grupos de discusión, con $\mathrm{R}$ para los relatos, con $\mathrm{F}$ cuando es un familiar, con $\mathrm{A}$ cuando es alumnado, con $\mathrm{P}$ cuando es profesorado y con $\mathrm{O}$ cuando es otros. El género se identifica con $\mathrm{M}$ para mujer y $\mathrm{H}$ para hombre.

Los resultados finales fueron consensuados y validados por el consejo asesor, celebrado en marzo de 2017 en la sede de la universidad de una de las coautoras de este artículo. En el consejo asesor se incluyó a personas pertenecientes a los colectivos investigados. Estas personas participan a título individual o como representantes de alguna organización. No obstante, se priorizó que hubiera representantes de los colectivos más vulnerables. Por 
ejemplo, se priorizó la participación de mujeres gitanas o pertenecientes a minorías culturales que no tienen titulación académica superior, que participan en formación de familiares y no forman parte de ninguna asociación. Finalmente, el consejo asesor estuvo formado por diez personas, entre estas, siete participantes en formación de familiares pertenecientes a minorías étnicas (cinco mujeres con niveles académicos iniciales, una estudiante universitaria gitana, un estudiante con título universitario gitano) $\mathrm{y}$ tres personas investigadoras, entre estas un investigador gitano.

\section{Resultados}

Se exponen los resultados a través de una síntesis representativa de las evidencias recogidas en el análisis de las entrevistas semiestructuradas, los grupos de discusión y los relatos de vida con orientación comunicativa de familiares, profesorado y voluntariado. La contribución principal que se ha identificado es que el voluntariado participante en la formación de familiares está reforzando las dinámicas de solidaridad ya existentes en estas escuelas. Estos están contribuyendo a crear más entornos de aprendizaje donde se establecen vínculos de ayuda mutua entre estos y las familias y entre las propias familias, reforzando el sentimiento de comunidad; así como, las interacciones igualitarias que fomentan relaciones de amistad, confianza y contribuyen al aumento de la motivación y las expectativas de las familias por su propia formación. A continuación, se presentan evidencias que respaldan estos resultados.

Uno de los beneficios que repercute en las familias, al incluir personas voluntarias en la formación de familiares, es que se crean más posibilidades de espacios de formación. En los casos analizados, el voluntariado está contribuyendo a que se puedan realizar más actividades de formación de familiares que, en su ausencia, no podrían ser llevadas a cabo. La inclusión de voluntariado en estas escuelas evita que se cancelen o no se lleguen a desarrollar actividades decididas por la comunidad por falta de recursos humanos. Ello contribuye a no crear un ambiente de desmotivación y pérdida de sentido, sino de motivación por parte de las familias que ven que aquello decidido se lleva a cabo. Esto se traduce en el aumento de la motivación de las familias por participar en estas actividades. Una profesora, que participa 
en una de las comisiones que gestiona la organización del voluntariado en su escuela, explicaba este impacto positivo en las madres. Esta lo explica con el ejemplo del compromiso del estudiantado universitario voluntario que se implica en las clases de graduado en educación secundaria.

Los voluntarios que tenemos, que nos mandan de la universidad, vienen fijo. Entonces las madres por pocas que fueran, si eran una o dos, ya había grupo. Entonces no había desánimo porque siempre había grupo. Entonces, las que iban estaban felices de ir; eso ha tirado de las demás porque ahora hay un grupo mucho más grande de madres. C2, E, P, M1

A su vez, el impacto del voluntariado se ve reforzado por las propias dinámicas de coordinación y organización en el funcionamiento diario de estas escuelas. Un familiar que participa en la escuela como voluntario explicaba que existe una importante tarea de coordinación y organización en la gestión del voluntariado. Ello repercute en generar un buen ambiente del que se contagia el voluntariado.

El buen ambiente que hay es palpable. Esto es fruto de que hay una buena coordinación. Hay un coordinador, que dice, tú vas a estar aquí, este voluntario va a estar allí, vais a trabajar juntos, vais a hacer esta tarea para la comunidad. Cada uno ya sabe, ya tiene su trabajo, y no hay más. C8, GD, F, H2

La organización democrática de estas escuelas contribuye también a que el voluntariado refuerce su compromiso porque siente que forma parte de la comunidad de aprendizaje. Al igual que el profesorado, el alumnado y las familias, el voluntariado puede participar en los espacios de decisión y gestión como, por ejemplo, las asambleas y las comisiones mixtas. El mismo padre hacía referencia a ese sentimiento de pertenencia a una comunidad como factor positivo para que la organización del voluntariado funcione bien y exista una responsabilidad por parte de estos.

Cada uno tiene una función aquí, pero todos somos comunidad de aprendizaje. [...]Cada uno tiene una posición y sabe cuál es su rol, 
su trabajo [...] Pero aquí funciona porque todos tienen un trabajo acometido, todos cumplen, todos respetamos. C8, GD, F, H2

Uno de los factores más destacados, por las personas participantes en el trabajo de campo, es que las interacciones entre el voluntariado y las familias se dan en el marco de una relación igualitaria, cercana y de creencia por parte del voluntariado en las capacidades de todas las personas. Ello promueve que las propias familias tengan altas expectativas sobre sus propios procesos de aprendizaje. Un profesor de una de estas escuelas, el cual ha tenido experiencia previa como voluntario en otros proyectos educativos fuera de su escuela, señalaba qué marca la diferencia del voluntariado en su escuela. Los que marca la diferencia según este profesor es el vínculo cercano que se establece entre las personas voluntarias y las familias. Este elemento lleva a que en la formación de familiares no sólo se tenga en cuenta las necesidades formativas de las familias, sino que también se fomenten altas expectativas respecto a su formación.

Yo he sido voluntario trabajando en clases de castellano con inmigrantes o con discapacidad y la diferencia (con su escuela) que más he notado es la relación directa que tiene (el voluntariado) con (las familias), que forman parte de algo. La otra formación es... hay un objetivo, claro, que es que aprendan castellano o que tengan una actividad de ocio, son objetivos concretos. Pero aquí además de ese objetivo concreto de la necesidad (formativa) que tiene el familiar, también hay un objetivo más comunitario de fondo, más de crear vínculos, de crear expectativas. C8, GD, P, H4

Esas interacciones entre el voluntariado y las familias basadas en las altas expectativas repercuten en la mejora del proceso de aprendizaje de estas. Así lo destaca una de las madres marroquíes de otra escuela, al hacer referencia a la diferencia que ella ha encontrado entre la formación de familiares que realiza en el centro de sus hijos y la que realizaba antes en una escuela de educación de personas adultas. En la escuela de personas adultas no existía la figura de la persona voluntaria y la relación con el profesorado era jerárquica y autoritaria. En las actividades de formación de familiares en la escuela de sus hijos existe un tipo de relación tanto con el profesorado como con el voluntariado que, según esta madre, le permite aprender más. 
Los voluntarios son buenísimos. Son gente que nos entiende y nosotros los entendemos a ellos. Están haciendo una labor muy buena. [...] No hay caras serias y esto hace que el ambiente sea más bueno. En Vitoria te sentabas en una mesa y "hazme un dictado, hazme esto, hazme lo otro y no copies". [...] Aprendes más de esta manera (como lo hacen en la escuela de sus hijos) porque estás con los profesores, luego estás con la gente que viene a ayudarte. C6, E, F, M1

Esta evidencia también se ha identificado en gran medida en los relatos de las personas voluntarias. Concretamente, un voluntario en la escuela de la madre de la cita anterior comenta la misma diferencia entre el tipo de relación que se establece con las personas participantes que la que se establece en una escuela de personas adultas en la que ha impartido clases. El voluntario afirma que en la escuela de personas adultas cada uno "va a lo que va" sin que se establezca una relación de cercanía. En cambio, en la escuela de infantil y primaria, donde se imparte formación de familiares, la relación es más afectiva y de confianza. Esto lleva también a una mayor motivación por participar tanto de la persona voluntaria como de las familias.

Para mí lo positivo es que, y lo comparo con las otras clases que veo, en la EPA (Educación de Personas Adultas), en las clases que doy (en la escuela de adultos) lo que no se establece es esta relación yo diría afectiva que se establece aquí (en la formación de familiares en la escuela de educación infantil y primaria). Y esto es para mí muy positivo, muy interesante. Y allí no, allí es más cada uno va a lo que va, es otra historia. Aquí tienes otra motivación. C6, GD, O, H1

Un aspecto para destacar es que el buen ambiente que se vive en la formación de familiares, de las escuelas que se han estudiado, no es porque haya voluntariado sino por el tipo de interacciones que el voluntariado promueve. El voluntariado acaba incorporando las interacciones solidarias que se viven en los diferentes entornos de aprendizaje o de decisión y gestión de las escuelas que son Comunidades de Aprendizaje. Por ejemplo, la persona voluntaria promueve que las propias personas participantes en la formación de familiares se ayuden entre ellas. Ante la necesidad de una 
explicación, el voluntario responsable de aquella actividad no es siempre quien responde, sino que promueve que la persona participante que ya tiene ese conocimiento lo comparta con las demás. Una de las voluntarias explicaba en su relato que cuando las personas participantes se ayudan entre ellas a comprender o resolver una actividad llegan a entenderlo mejor que cuando lo explicaba la persona voluntaria, como se puede apreciar en la siguiente cita.

Que se expliquen ellos también. El tema del aprendizaje dialógico, esto les sirve un montón. Y hay veces que tú te empecinas en explicar una cosa y claro y dices ¿cómo no lo ven? Pero igual si Nieves se lo explica a Dina y Ramón se lo explica a Miguel, ellos lo ven más fácil. C8, GD, O, M1

La solidaridad y ayuda mutua acaba normalizándose entre los y las familiares participantes. Este es un aspecto destacado por ellas mismas como factor que contribuye a un mejor aprendizaje. Una de las madres participantes así lo expresa: A veces nos preguntamos y el que más sabe pues te explica (C6, E, F, M2). Otro familiar participante hacía referencia a que ello contribuye a cohesionar el grupo, identificándolo como una gran familia.

Esto es como una gran familia. Nos ayudamos unos a los otros. [...] Lo que igual yo no entiendo, pues esta mujer lo entiende y me lo explica, o al revés se lo explico yo a ellas. Nos ayudamos. C6, GD, $\mathrm{F}, \mathrm{H} 1$

En tres de las escuelas estudiadas desde hace varios años mantienen un grupo de castellano oral para madres marroquíes dinamizado por voluntarias. Desde hace unos años algunas de las voluntarias también son mujeres marroquíes madres de la escuela. Como ellas mismas señalan, en este grupo no solo aprenden, sino que fruto de esa solidaridad, apoyo mutuo y relaciones igualitarias se crean lazos de amistad. Una de las madres afirmaba: aprendes muchas cosas, conoces a las compañeras, haces amistad con ellas, con las profesoras (voluntarias) también (C1, GD, F, M1). Una de las voluntarias marroquíes destacaba ese buen ambiente haciendo referencia a que "se crea 
un espacio bonito" de amistad que repercute también en el aumento de la motivación por participar.

Se crea un espacio muy bonito. Ahora mismo lo que estamos viviendo es que es muy dulce. Vienen con mucha predisposición y con ganas. Ninguna viene a lucirse o intentar destacar, porque todas se ven que entre ellas son las mejores amigas y que yo también soy su mejor amiga. [...] Ellas vienen con ganas de encontrarse. C4, E, $\mathrm{O}, \mathrm{M} 1$

El hecho de que algunas de las voluntarias también sean marroquíes ha facilitado el proceso de formación de estas mujeres. Así lo explicaba una de las madres participantes.

El hecho de que la voluntaria hable nuestro idioma nos ha facilitado muchísimo. Una persona que habla otro idioma no te va enfocar la formación según la podamos captar. Pero ella, al ser de la misma cultura, al hablar la misma lengua que nosotras, sabe la necesidad que tenemos y nos da lo que nosotras necesitamos porque sabe cómo sacaremos el máximo provecho. C4, GD, M2.

En otra de las escuelas que tiene un grupo de formación para madres marroquíes, para facilitar la participación de estas en las actividades se ha permitido que asistan con los hijos y las hijas más pequeños aún no escolarizados. Pero ellas, posteriormente decidieron que podían concentrarse más en la actividad si asistían sin niños y niñas. El ambiente de solidaridad que se ha creado en el grupo, promovido por las voluntarias, ha repercutido a que las relaciones entre ellas también sean solidarias. Las madres han auto-gestionado una red solidaria para el cuidado de los y las más pequeños. Entre ellas, hacen turnos para quedarse al cargo de estos mientras las otras participan en las actividades de formación. En uno de los grupos de discusión con el profesorado de esta escuela, se reflexionaba sobre qué factores facilitaban la participación de estas madres en las actividades de formación de familiares. Un factor clave que señalaron fue la posibilidad de poder tener un espacio en el que ellas pudieran dejar a los niños y niñas más pequeños para que los cuidaran mientras participaban en la formación. El profesorado explicaba cómo mientras se buscan 
soluciones para conseguir ese espacio, ellas ya se habían autoorganizado para solucionarlo momentáneamente.

Profesor 1. Una de las cosas que les facilitaría a ellas asistir con más regularidad, que es lo que decían que a lo mejor no hacían, sería la posibilidad de dejar (a los niños). Muchas de ellas tienen niños más pequeños que no están en edad escolar. C5, GD, P, H1

Profesora 4. Bueno esto lo resuelven, una mamá se queda con ellos. C5, GD, P, M4

Profesora 2. Han montado una guardia entre ellas, se turnan. Empezaron trayendo a los niños y, luego, ya empezaron montando el sistema entre ellas. Un sistema guardería. C5, GD, P, M2

Las dinámicas solidarias promovidas en la formación de familiares tienen un impacto más allá de los propios grupos de formación. Las familias que participan en la formación acaban rompiendo las barreras que a menudo se crean entre estas y la escuela, sobre todo, en familias no académicas pertenecientes al pueblo gitano o de origen inmigrante. El profesorado participante en el estudio ha valorado la formación de familiares como un factor transformador que ha acercado a las familias a la escuela. Uno de los profesores entrevistados así lo señalaba:

El hecho de que la familia esté dentro del centro ya es positivo porque rompe con la barrera: la familia es una cosa y la escuela otra. (C1, W, P, H2).

Las familias sienten ahora la escuela como un espacio que también les pertenece. En uno de los grupos de discusión con profesorado, la directora de otra de las escuelas ponía de manifiesto cómo ahora las madres marroquíes han ganado seguridad para entrar en la escuela y relacionarse en confianza con el profesorado, el voluntariado y las otras personas del equipo.

El cómo ellas vienen ahora y te preguntan y quieren saber. Ellas ahora no tienen ningún reparo en llegar a dirección y preguntarte "¿qué pasa con los libros? ¿Qué vais a hacer con esta excursión?", y se las ve relajadas cuando vienen a consultarte y cuando vienen a preguntarte. Y entran y salen, y lo mismo en la secretaría. En esto 
se ha notado que ellas han ganado cierta seguridad y ya no les importa venir al cole. C5, GD, P, M2

\section{Conclusión}

El voluntariado en la formación de familiares implementada en las escuelas objeto de esta investigación ha contribuido a fortalecer las dinámicas solidarias que ya existían como principio organizativo en estas. Estas dinámicas solidarias, promovidas por el voluntariado, parten de un compromiso con las familias y la escuela. Ese compromiso se concreta en una actitud del voluntariado igualitaria, cercana que cree en las capacidades de todas las personas y que fomenta las altas expectativas en las familias. La relación igualitaria entre el voluntariado y las familias contribuye a crear un ambiente en los grupos de formación de familiares que facilita las relaciones solidarias, de confianza y de amistad entre las propias familias. Asimismo, tal y como se ha destacado en la introducción, la mejora de las interacciones, en este caso a partir de una gran diversidad de voluntariado, de los niños y niñas de grupos vulnerables contribuye a le mejora del rendimiento académico (Bonell y Ríos, 2014; Garcia-Carrion, Molina-Luque, y MolinaRoldan, 2018). Todo ello repercute en un aumento de la motivación de las familias por participar en la formación de familiares y en la mejora de su proceso de aprendizaje.

Los resultados de esta investigación comportan una implicación social al aportar conocimiento sobre qué tipo de actitud del voluntariado contribuye a una formación de familiares de éxito. Como es sabido por investigaciones previas, la formación de familiares que responde a las necesidades e intereses de las familias tiene un impacto en la mejora de los resultados académicos de los niños y las niñas y en la mejora del ambiente escolar; superando así los determinismos educativos que condenan al fracaso escolar a los niños y niñas de las familias más vulnerables (Garcia-Yeste, Morlà, y Ionescu, 2019; Flecha y Soler, 2013). Por ello, el fin último de la inclusión de voluntariado en la formación de familiares en estas escuelas es que esa formación sea de éxito, contribuyendo así a aumentar el nivel educativo de las familias y, a su vez, tener un impacto en la mejora de los resultados educativos de los niños y niñas, así como en la mejora de la convivencia. 
REMIE - Multidisciplinary Journal of Educational Research, 9(2) 163

Se muestra así, un ejemplo del valor transformador de la solidaridad a través de la acción del voluntariado en comunidades de aprendizaje, como una herramienta clave en la mejora de la convivencia y los resultados en contextos educativos con grupos vulnerables.

\section{Referencias}

Aubert, A.; Flecha, A.; García, C.; Flecha, R. Y Racionero, S. (2008). Aprendizaje Dialógico en la Sociedad de la Información. Barcelona: Hipatia Press.

Aubert, A., Molina, S., Shubert, T., Vidu, A. (2017). Learning and inclusivity via Interactive Groups in early childhood education and care in the Hope school, Spain. Learning, Culture and Social Interaction, 13, 90-103. doi: 10.1016/j.lcsi.2017.03.002

Baudelot, C., y Establet, R. (1976). La educación capitalista en Francia. Madrid: Siglo XXI.

Bonell, L., y Ríos, O. (2014). Participation and family education in school:

Successful educational actions. Studies in the Education of Adults, 46(2), 177-191. doi: 10.1080/02660830.2014.11661665

Boucher, ML. (2016). More Than an Ally: A Successful White Teacher Who Builds Solidarity with His African American Students. Urban Education, 51(1), 82-107. doi: 0.1177/0042085914542982

Bourdieu, P., y Passeron, J. C. (1996). La reproducción. Elementos para una teoría del sistema de enseñanza. México D.F: Distribuciones Fontamara.

Bowles, S., y Gintis, H. (1985). La instrucción escolar en la América capitalista. Madrid: Siglo XXI.

Coleman, J., Campbell, E., Hobson, C., McPartland, F., Mood, A., Weinfeld, F., et al. (1966). Equality of educational opportunity. Washington, DC: U.S. Government Printing Office.

Comisión Europea (2006) Eficiencia y equidad en los sistemas europeos de educación y formación. COM (2006) 481 final. Bruselas, 8.9.2006. Recuperado de: http://eur-lex.europa.eu/legalcontent/ES/TXT/PDF/?uri=CELEX:52006DC0481\&from=EN 
164 Garcia, Ruíz, \& Comas -Formación de Familias y Voluntariado

Comisión Europea (2010) Una estrategia para un crecimiento inteligente, sostenible e integrador. $\mathrm{COM(2010)} 2020$ final. Bruselas, 3.3.2010. Recuperado de: http://eur-lex.europa.eu/legalcontent/ES/TXT/PDF/?uri=CELEX:52010DC2020\&from=ES

Cheney, G., Santa Cruz, I., Peredo, A. M., y Nazareno, E. (2014). Worker cooperatives as an organizational alternative: Challenges, achievements and promise in business governance and ownership. Organization, 21(5), 591-603. doi:

$10.1177 / 1350508414539784$

De Botton, L. (2015). The instrumental dimension in learning communities. Intangible Capital, 11(3), 350-371. doi: 10.3926/ic.659

Dearing, E., Kreider, H., Simpkins, S., y Weiss, H. B. (2006). Family involvement in school and low-income children's literacy: Longitudinal associations between and within families. Journal of Educational Psychology, 98(4), 653-664. doi: 10.1037/00220663.98.4.653

Diez, J., Gatt, S., y Racionero, S. (2011). Placing Immigrant and Minority Family and Community Members at the School's Centre: the role of community participation. European Journal of Education, 46(2), 184-196. doi: 10.1111/j.1465-3435.2011.01474.x

Edmonds, R. et al. (1973) A Black Response to Christopher Jenck's Inequality and Certain Other Issues. Harvard Educational Review, 43(1), 76-91.

Epstein, JL., y Sheldon, SB.(2002). Present and accounted for: improving student attendance through family and community involvement. The Journal of Educational Research, 95(5), 308-318. doi:

10.1080/00220670209596604

Epstein, JL (1996). Improving School-Family-Community Partnerships in the Middle Grades. Middle School Journal, 28(2), 43-48. doi: 10.1080/00940771.1996.11494440

Epstein, JL., y Sanders, MG. (2006). Prospects for change: preparing educators for school, family, and community partnerships. Peabody Journal of Education, 81(2), 81-120. doi: 10.1207/S15327930pje8102_5

Epstein, JL. (2016). Searching for equity in education: Finding school, family, and community partnerships. En A. R. Sadovnik y R. W. 
REMIE - Multidisciplinary Journal of Educational Research, 9(2) 165

Coughlan (eds.), Leaders in the Sociological Study of Education (6985). Springer. Recuperado de:

https://link.springer.com/book/10.1007/978-94-6300-717-7

European Commission (2011). Added value of reserach, Innovation and

Science portfolio. European Commission - MEMO/11/520

19/07/2011 Recuperado de: http://europa.eu/rapid/press-

release_MEMO-11-520_es.htm?locale=fr

Flecha, R., y Soler, M. (2013). Turning difficulties into possibilities: engaging Roma families and students in school through dialogic learning. Cambridge Journal of Education, 43(4), 451-465. doi: 10.1080/0305764X.2013.819068

Flecha, R., y Ngai, P. (2014). The challenge for Mondragon: Searching for the cooperative values in times of internationalization. Organization, 21(39), 666-682. doi:

10.1177/1350508414537625

Flecha, R. (2015). Successful Educational Actions for Inclusion and Social Cohesion in Europe. Springer Publishing Company. doi: 10.1007/9783-319-11176- 6

Flecha, R. (2017). Social Impact of Community-Based Educational Programs in Europe. Oxford Research Encyclopedia of Education. Oxford: Oxford University Press.

Garcia, R., Girbes, S., y Gomez, A. (2015). Promoting children's academic performance and social inclusion in marginalized settings: Family and community participation in interactive groups and dialogic literary gatherings. In L. D. Hill y F. J. Levine (Eds.), World education research yearbook 2015 (pp. 1-7). New York: Routledge.

Garcia-Carrion, R., Molina-Luque, F., y Molina-Roldan, S. M. (2018). How do vulnerable youth complete secondary education? The key role of families and the community. Journal of Youth Studies. 27(14), 701716. doi: 10.1080/13676261.2017.1406660

Garcia-Yeste, C., Gairal, R., Munte, A., y Plaja, T. (2018). Dialogic literary gatherings and out-of-home child care: Creation of new meanings through classic literature. Child \& Family Social Work, 23(1), 62-70. doi: $10.1111 / \mathrm{cfs} .12384$

Garcia-Yeste, C., Morla, T., y Ionescu, V. (2018). Dreams of Higher Education in the Mediterrani School through Family 
166 Garcia, Ruíz, \& Comas -Formación de Familias y Voluntariado

Education. Frontiers in Education, 3(79). doi:

10.3389/feduc.2018.00079

Gómez, A., Padrós, M., Ríos, O, Mara, L.C., \& Pukepuke, T. (2019)

Reaching Social Impact Through Communicative Methodology.

Researching With Rather Than on Vulnerable Populations: The Roma

Case. Frontiers in Education, 4(9). doi: 10.3389/feduc.2019.00009

Gomez, A., Puigvert, L., y Flecha, R. (2011). Critical communicative methodology: informing real social transformation through

research. Qualitative Inquiry, 17(3), 235-245. doi:

10.1177/1077800410397802

Gómez, J., Latorre, A., Sánchez, M., and Felcha, R. (2006). Metodología Comunicativa Crítica. Barcelona: Hipatia.

Hermansen, J. (2016). Intangible activities-the prevalence of informal helping in Denmark. Journal of Civil Society, 12(4), 380-393. doi: 10.1080/17448689.2016.1235676

Jasis, PM., Ordoñez, I., Jasis, R. (2012). Latino parent involvement:

Examining commitment and empowerment in schools. Urban

Education: 47(1), 65-89. Recuperado de:

http://uex.sagepub.com/content/47/1/65.full.pdf

Llopis, A., Villarejo, B., Soler, M., y Álvarez, P. (2016). (Im)Politeness and interactions in Dialogic Literary Gatherings. Journal of Pragmatics, 94, 1-11. doi: 10.1016/j.pragma.2016.01.004

Mcgovern, F., y Devine, D. (2016). The care worlds of migrant children exploring inter-generational dynamics of love, care and solidarity across home and school. Childhood-A Global Journal of Child Research, 23(1), 37-52. doi: 10.1177/0907568215579734 Padros, M., y Flecha, R. (2014). Towards a Conceptualization of Dialogic Leadership. International Journal of Educational Leadership and Management, 2(2), 207-266. doi: 10.4471/ijelm.2014.17

Pulido, C., Elboj, C., Campdepadros, R., y Cabre, J. (2014). Exclusionary and Transformative Dimensions Communicative Analysis Enhancing Solidarity Among Women to Overcome Gender Violence. Qualitative Inquiry, 20(7), 889-894. doi: 10.1177/1077800414537212

Pulido-Rodríguez, M., Amador, J., y Alonso-Rodrigo, E. (2015). Manuel, recovering the sense of the democratic movement through 
REMIE - Multidisciplinary Journal of Educational Research, 9(2) 167

living solidarity in dialogic literary gatherings. Qualitative Inquiry, 21(10), 851-857. doi: 10.1177/1077800415614027

Ramis-Salas, M. (2015). Plurality and equality in the Learning

Communities. Intangible Capital, 11(3), 293-315. doi: 10.3926/ic.580

Sorde, T., Serradell, O., Puigvert, L., y Munte, A. (2013). Solidarity

networks that challenge racialized discourses: The case of Romani immigrant women in Spain. European Journal of Women's Studies, 21, 87-102. doi: 10.1177/1350506813510425

Valero, D., Redondo-Sama, G. \& Elboj, C. (2018). Interactive groups for immigrant students: a factor for success in the path of immigrant students. International Journal of Inclusive Education, 22(7), 787-802. doi: 10.1080/13603116.2017.1408712

Valls, R., y Kyriakides, L. (2013). The power of interactive groups: how diversity of adults volunteering in classroom groups can promote inclusion and success for children of vulnerable minority ethnic populations. Cambridge Journal of Education, 43(1), 17-33. doi: 10.1080/0305764X.2012.749213

Youngblood, JH. (2017). Intangible benefits of volunteering with the Heart Rhythm Society. Heart Rhythm, 14(1). doi: 10.1016/j.hrthm.2016.10.023 
Carme Garcia Yeste es profesora junto al Departamento de Pedagogía de la Facultat de Ciències de l'Educació i Psicologia, Universitat Rovira i Virgili, Catalunya, España.

\section{Orcid: http://orcid.org/0000-0001-9717-8021}

Laura Ruiz Eugenio es Investigadora Ramón y Cajal. Departamento de Teoría e Historia de la Educación. Universitat de Barcelona, Catalunya, España.

\section{Orcid: http://orcid.org/0000-0002-2262-1663}

Miquel Àngel Comas es profesor colaborador junto a la Facultad de Educación de la Universidad Internacional de la Rioja, España.

\section{Orcid: https://orcid.org/0000-0002-0104-5011}

Contact Address: Carme Garcia Yeste. Departamento de Pedagogía de la Facultat de Ciències de l'Educació i Psicologia, Universitat Rovira i Virgili, Catalunya, España 